\title{
Búsqueda Activa de Individuos con Tuberculosis Pulmonar y Extrapulmonar en Calarcá-Quindío, Colombia-2005
}

\author{
Detecting active tuberculosis in Calarcá-Quindío, Colombia, \\ during 2005
}

\author{
Nelson E. Arenas ${ }^{1}$, Elizabeth Torres ${ }^{1}$, Clara J. Durango ${ }^{1}$, Laura I. Cuervo ${ }^{1}$, Sandra \\ M. Coronado ${ }^{1}$ y Arley Gómez ${ }^{1,2}$ \\ 1 Centro de Investigaciones Biomédicas. Facultad de Ciencias de la Salud, Universidad del Quindío. \\ Armenia, Colombia. \\ 2 Unidad de Enfermedades infecciosas y Medicina Tropical. Facultad de Medicina. Universidad del \\ Rosario. Bogotá D.C. Colombia. arley.gomez24@urosario.edu.co
}

Recibido 14 Agosto 2007/Enviado para Modificación 20 Marzo 2008/Aceptado 22 Abril 2008

\section{RESUMEN}

Objetivo Describir características socio demográficas en una muestra de 195 pacientes sintomáticos respiratorios y 18 pacientes tuberculosos de Calarcá - Quindío, Colombia en el 2005.

Metodología Se realizó búsqueda activa de tuberculosos en tres zonas urbanas, la cárcel del municipio de Calarcá y el Hospital Universitario San Juan de Dios. Previo ingreso al estudio los pacientes diligenciaron una encuesta y un consentimiento informado. A cada paciente se le tomaron muestras de esputo para baciloscopia y cultivo en medio de Ogawa-Kudoh. Cuando se observó crecimiento se realizó identificación fenotípica por pruebas bioquímicas y genotipificación por spoligotyping. Resultados Se identificaron 195 pacientes sintomáticos respiratorios y 18 (9,2\%) pacientes tuberculosos, de ellos el $78 \%$ corresponde al género masculino y $22 \%$ al femenino con una edad promedio para ambos sexos de 40 años. Se procesaron un total de 174 muestras de esputo, 21 lavados bronco alveolares, una muestra de orina, una muestra de ganglio cervical y un liquido pleural. El $77 \%$ de las muestras analizadas fueron positivos por examen directo y cultivo, $16,7 \%$ por cultivo y el 5,5\% por baciloscopia únicamente. El promedio de baciloscopias por paciente fue 2,3. La prueba de spoligotyping realizada evidenció la presencia de los genotipos H1, T1, H3 y LAM9.

Conclusión Los resultados obtenidos demuestran la importancia de realizar simultáneamente baciloscopia y cultivo para aumentar la detección de pacientes bacilíferos sintomáticos respiratorios, al igual que en el seguimiento y control de estos pacientes. Se demostró la presencia de cuatro genotipos diferentes de Mycobacterium tuberculosis en Calarcá.

Palabras Clave: Tuberculosis, transmisión, signos y síntomas respiratorios, (fuente: DeCS, BIREME). 


\begin{abstract}
Objective Describing socio-demographic characteristics in a population of 195 respiratory symptomatic and 18 tuberculosis patients from Calarcá-Quindío, Colombia during 2005.

Methods An active search for individuals having symptomatic tuberculosis was carried out in three urban areas, Calarca's municipal prison and the San Juan de Dios teaching hospital. Prior to entering the study, the patients were required to fill out an application form and sign an informed consent form. Sputum samples were taken from each patient for bacilloscopy and culture in Ogawa-Kudoh medium. Phenotypic and genotypic identification were made when growth was observed by using conventional and molecular methods.

Results $18(9,2 \%)$ out of 195 individuals having respiratory symptoms were diagnosed as having tuberculosis. $78 \%$ were male and $22 \%$ female; the average age was 40 for both genders. 174 sputum, 21 bronchial lavage, one urine, one cervical ganglia and pleural liquid sample were analysed; $77 \%$ proved positive by direct smear test and culture, $16,7 \%$ by culture and only $5,5 \%$ by direct smear test. Bacilloscopy average per patient was 2,3. The presence of H1, T1, H3 and LAM9 genotypes was demonstrated by using the spoligotyping test.

Conclusions The results confirmed the importance of using both direct smear test and/or culture for improving the early detection of pulmonary tuberculosis, its followup and control. The existence of four different Mycobacterium tuberculosis genotypes in Calarcá was established.
\end{abstract}

Key Words: Tuberculosis, respiratory symptoms, signs and symptoms, respiratory (source: MeSH, NLM).

L

a tuberculosis (TB) es una enfermedad reconocida como una causa importante de incapacidad y muerte de personas en muchas zonas del mundo. La infección inicial suele ser asintomática y las lesiones por lo general se curan y no dejan alteraciones residuales. Aproximadamente el $95 \%$ de las personas infectadas inicialmente entran a esta fase de latencia, a partir de la cual existe el peligro permanente de reactivación (1). En el 5 \% de los casos restantes, la infección inicial puede evolucionar de manera directa hasta culminar en TB pulmonar o causar afección miliar, meníngea o de localización extrapulmonar. En los niños se desconoce la prevalencia real de la enfermedad dada la dificultad del diagnostico, en los adultos jóvenes es más frecuente que en otros grupos y en toda la población afectada en general, puede alcanzar consecuencias y secuelas graves si no es detectada y tratada a tiempo (2).

En el Quindío la tasa de incidencia establecida para TB es de 32,8 casos por 100000 habitantes por lo cual el departamento ha sido clasificado como de alto riesgo comparado con el nivel nacional cuyo reporte para el año 2004 fue de 25,7 
casos por 100000 habitantes; paralelamente en el municipio de Calarcá se reportaron 36 casos de TB incluidas las formas pulmonares y extrapulmonares representando una tasa de 44,4 casos por 100000 habitantes, una de las tasas más altas a nivel regional y superior a la media nacional en el 2004, (3) lo cual evidencia que la tuberculosis sigue siendo un problema en este municipio. Es importante resaltar que en el mismo año se diagnosticaron 198 casos nuevos de tuberculosis con baciloscopia positiva en el departamento de Quindío, equivalentes a una tasa de 32,8 casos por 100 mil habitantes (3); dado que los pacientes bacilíferos son detectados frecuentemente en el tercer nivel, ello podría explicar la transmisibilidad y alta incidencia de la enfermedad en la región; además, el diagnostico tardío empeora el pronóstico para el paciente y en algunas ocasiones las cepas aisladas son resistentes a los antituberculosos.

Esta situación pone de presente la importancia de mejorar la búsqueda, captación y seguimiento del paciente sintomático respiratorio y aumentar la sospecha de TB por parte del cuerpo medico a fin de aumentar la detección temprana de la enfermedad; sin embargo, una considerable proporción de la población del eje cafetero es fluctuante y las constantes migraciones se constituyen en un grave problema para controlar la transmisión y realizar el seguimiento de los pacientes tuberculosos como indigentes, en los cuales suelen aumentarse las complicaciones por abandono del tratamiento y dado los factores socio-económicos asociados a su calidad de vida pueden contribuir a aumentar la cadena de transmisión por sus actividades ambulatorias.

En Colombia se han realizado algunos estudios respecto a la prevalencia de sintomáticos respiratorios (SR) y pacientes con diagnostico de TB en algunos municipios como Medellín, (4) Mitú, (5) Cubará(6) y el departamento del Guaviare (7) en los cuales se ha identificado la necesidad de implementar estrategias para mejorar la detección de casos y el diagnostico tardío, acercándose a un mejor conocimiento de la situación de la TB en el país (8). Actualmente en la región no existen estudios publicados donde se haya determinado la prevalencia de TB, aun conociendo la relevancia de este tipo de estudios en el contexto epidemiológico y de la salud pública. En este contexto, otros estudios han demostrado la necesidad de conocer las tasas exactas de TB y las características sociodemográficas de la población afectada para conocer el comportamiento de la enfermedad a través del tiempo y así poder fortalecer las intervenciones en el programa de control, dirigiendo acciones prioritarias a los grupos con mayor riesgo (9).

Ante la problemática local, la presente investigación tuvo como objetivo identificar algunas características socio demográficas de una población de 195 SR y 
18 pacientes con diagnostico de TB en el municipio de Calarcá durante los años 2004 y 2005.

\section{MATERIALES Y MÉTODOS}

Tipo de estudio y población referencia

Se presenta un estudio descriptivo el cual incluye el análisis de frecuencias y porcentajes de las variables sociodemográficas de los pacientes SR y con diagnóstico presuntivo de TB captados en el Centro de Investigaciones Biomédicas provenientes del Hospital Universitario San Juan de Dios de Armenia y el municipio de Calarcá durante el periodo de octubre 2004 a junio 2005 por búsqueda activa en la Cárcel del municipio y en algunos barrios de bajos estratos socioeconómicos del municipio donde existían casos reportados de TB a la secretaría de salud del municipio. Se realizó la encuesta a los contactos del mismo núcleo familiar o unidad residencial y a los vecinos de la misma cuadra donde se encontraban los casos índices.

Los pacientes SR fueron definidos como las personas que manifestaron tener tos y expectoración por más de quince días y algún síntoma adicional compatible con sospecha de TB pulmonar como fiebre, pérdida de peso, sudoración (diaforesis) y/o tos con sangre (Hemoptisis).

Área de estudio

El municipio de Calarcá tiene una superficie de $219 \mathrm{Km}^{2}$ es la segunda ciudad en importancia del Departamento del Quindío y se encuentra al occidente de Armenia la capital del departamento. Cuenta con una población de 82400 habitantes distribuida en 95 barrios incluyendo urbanizaciones e invasiones en el área urbana y 27 veredas en el área rural (3). El municipio tiene 3 centros de salud de atención primaria y un Hospital de segundo nivel (Hospital La Misericordia), los casos de mayor complejidad son remitidos al tercer nivel (Hospital Universitario San Juan de Dios de Armenia), para su valoración y seguimiento por personal especializado.

Se implementaron brigadas de captación de pacientes en zonas de bajo nivel socio económico, específicamente en los barrios Lincon, Balcones de la Villa, Hogar del anciano y en la de prisión Peñas Blancas, en razón de ser las áreas con mayor incidencia de la TB según información consignada en la Secretaria de Salud del Municipio; a los participantes se les diligenció la respectiva encuesta epidemiológica y se practicó la toma de muestra en recipientes plásticos estériles. 
Métodos de recolección de información y muestras

La información recolectada a través de la encuesta epidemiológica realizada a cada uno de las pacientes incluyó variables demográficas como edad, género, tipo de afiliación al sistema general de seguridad social en salud, procedencia, antecedentes a tratamiento antituberculoso, síntomas y tipo de muestra. Dicha información fue almacenada en bases de datos en hojas de cálculo de Excel y analizada utilizando el programa EPI info 2002 (10).

A los pacientes incluidos en este estudio se les diligenció un consentimiento informado, en el cual se les suministro información detallada acerca del procedimiento y sus beneficios en términos diagnósticos, terapéuticos y económicos.

Pruebas fenotípicas de identificación y genotipificación

A todas las muestras se le practicaron pruebas diagnósticas de laboratorio: tinción Ziehl Nielsen realizando un análisis paralelo acerca de la calidad de la muestra, cultivo por la técnica de Ogawa modificada por Kudoh y en caso de presentar crecimiento bacteriano, la identificación de Mycobacterium tuberculosis se realizó utilizando las pruebas bioquímicas convencionales como: pirazinamidasa, niacina, nitratos, ureasa, TCH, susceptibilidad a estreptomicina, preferencia por oxígeno e inspección de la morfología de las colonias (11).

A las cepas viables de Mycobacterium tuberculosis con buen crecimiento en medio de cultivo, se les realizó extracción de $\mathrm{ADN}$ que fue almacenado a $4^{\circ} \mathrm{C}$ hasta su utilización para la caracterización genotípica mediante la técnica de Spoligotyping (12,13).

\section{RESULTADOS}

Características socio-demográficas de SR

Durante el estudio se captaron 195 individuos SR de los cuales (74,8\%) pertenecía al género masculino y un (23,2 \%) al femenino. En total se analizaron 174 muestras de esputo, 21 lavados broncoalveolares, 1 muestra de orina, 1 muestra de ganglio cervical y 1 muestra de liquido pleural a las cuales se les realizaron 454 baciloscopias y 195 cultivos. La tasa de baciloscopias por paciente fue de 2,3 y la positividad fue de 3,9\% para la población estudiada.

Un 89,7 \% de los individuos SR incluidos en el estudio manifestaron no haber recibido tratamiento contra TB previamente, sólo un mínimo porcentaje (5.6 \%), declararon haber recibido tratamiento antituberculoso y para el 4,7 \% de la población restante no se obtuvo un registro de sus antecedentes de tratamiento antituberculoso. 
Detección de pacientes tuberculosos

Se diagnosticaron 18 pacientes positivos para TB principalmente de tipo pulmonar. La proporción de estos pacientes tuberculosos mantuvo una tendencia similar a la presentada en la búsqueda de SR respecto a la predominancia del género masculino; 4 mujeres y 14 hombres fueron diagnosticados y sus edades oscilaron entre los 15 y 49 años para la mayoría de la población (Tabla 1). La edad promedio para ambos sexos fue de 40 años y según los registros analizados, la mayor proporción de individuos correspondieron al nivel bajo (72,2 \%), en correspondencia con su afiliación al sistema general de seguridad social en salud.

Tabla 1. Edad y sexo en pacientes con diagnóstico de tuberculosis

\begin{tabular}{cccc}
\hline Años & Hombres & Mujeres & Total \\
\hline $0-14$ & 0 & 0 & 0 \\
$15-49$ & 11 & 3 & 14 \\
Mayor de 50 & 2 & 1 & 3 \\
Sin registro & 1 & 0 & 1 \\
\hline Total & 14 & 4 & 18
\end{tabular}

Entre los 18 pacientes con diagnóstico de TB el síntoma predominante fue la tos con expectoración por más de 15 días ( $88,8 \%$ ), seguido por perdida de peso (27,7 \%) y fiebre (22,2 \%), el diagnóstico fue confirmado por baciloscopia y cultivo en un $77 \%$ de los casos; sin embargo se presentaron 3 casos de pacientes positivos paucibacilares diagnosticados a partir de muestras de esputos que resultaron con baciloscopia negativa y cultivo positivo (Tabla 2).

Tabla 2. Baciloscopia y cultivo en pacientes tuberculosos, según antecedentes de tratamiento

\begin{tabular}{ccccc}
\hline \multirow{2}{*}{ Antecedentes } & \multicolumn{4}{c}{ Resultados } \\
\cline { 2 - 5 } & $\begin{array}{c}\text { Baciloscopia }(+) \\
\text { Cultivo }(-) \\
(\%)\end{array}$ & $\begin{array}{c}\text { Baciloscopia }(+) \\
\text { Cultivo }(+) \\
(\%)\end{array}$ & $\begin{array}{c}\text { Baciloscopia }(-) \\
\text { Cultivo }(+) \\
(\%)\end{array}$ & $\begin{array}{c}\text { Total } \\
(\%)\end{array}$ \\
\hline Sin tratamiento & $1(5,5)$ & $13(72,2)$ & $3(16,7)$ & $17(8,7)$ \\
Con tratamiento & 0 & $1(5,5)$ & 0 & $1(5,5)$ \\
\hline TOTAL & $1(5,5)$ & $14(77,7)$ & $3(16,7)$ & 18 \\
\hline
\end{tabular}

Tabla 3. Aspecto de las muestras de esputo de pacientes sintomáticos respiratorios del municipio de Calarcá

\begin{tabular}{lcc}
\hline Aspecto de la Muestra & Pacientes & Porcentaje (\%) \\
\hline Mucopurulenta & 85 & 18,7 \\
Mucosalival & 185 & 40,7 \\
Salival & 152 & 33,5 \\
Purulenta & 17 & 3,8 \\
Mucosa & 15 & 3,3 \\
\hline
\end{tabular}


El 94,4 \% de los pacientes con diagnostico de TB, manifestaron no haber recibido tratamiento antituberculoso, solo se presentó un caso de un paciente con antecedentes quimioterapéuticos con resultado positivo en la baciloscopia y el cultivo (Tabla 2). En el análisis de la calidad de la muestra se encontró que el 74,2 $\%$ de las muestras presentaban un aspecto entre muco salival y salival (Tabla 3) este hallazgo podría estar asociado a la baja incidencia de pacientes positivos para Mycobacterium tuberculosis a pesar de la búsqueda activa en sitios que podrían representar fuentes de contagio y alta incidencia para TB en el municipio de Calarcá.

Genotipificación de cepas de Mycobacterium tuberculosis

Se evaluaron cinco aislados y los patrones identificados fueron comparados con los reportados en la base de datos central SpolDB4 (14), demostrándose la presencia de los genotipos Harleem 1, (H1) Harleem 3 (H3), LAM9 y T1, este ultimo genotipo presente en dos cepas pero debido al bajo número de muestras caracterizadas por este método no pudimos establecer correlación con otras variables sociodemográficas o clínicas.

\section{DISCUSIÓN}

Cuando se compara la proporción de SR hallados en el municipio de Calarcá con prevalencias similares en otros municipios del país como la encontrada en el municipio de Cubará (Boyacá) con 179 SR en una población de 2865 habitantes, se estableció una mayor proporción de pacientes del sexo femenino en Calarcá con respecto al estudio en Boyacá; sin embargo se debe tener en cuenta que la población estudiada en el municipio de Cubará era indígena y por ello las condiciones predisponentes tanto la incidencia como la prevalencia en enfermedades como la TB pueden ser diferentes y asociadas con factores socio-económicos, barreras geográficas, de salud y culturales propios, que facilitan la diseminación de la enfermedad y dificultan su tratamiento (6). No obstante, otros estudios reportan factores que pueden estar relacionados con el menor registro de SR entre géneros; como el hecho de que las mujeres se automedican con mayor frecuencia a modo de acción primaria en salud y en consecuencia acuden menos a la consulta que los hombres (15).

La mayor proporción de SR del género masculino en este estudio es resultado de la búsqueda de pacientes en las cárceles de hombres de Calarcá, no obstante lo anterior también se aplica para la población captada únicamente en la comunidad e instituciones de salud. Es importante destacar que la ausencia de pacientes con resultado positivo por examen directo o por cultivo no implica la 
inexistencia de la enfermedad. Así mismo, consideramos que este hecho no debe desalentar la búsqueda de SR y casos de TB en prisiones, ya que los individuos portadores asintomáticos y los sintomáticos respiratorios no diagnosticados y por ende no tratados representan focos de infección en la medida que el hacinamiento aumenta el riesgo de contagio de la enfermedad(16). Además en las prisiones de esta región las condiciones sanitarias representan un factor de riego para la transmisión de TB.

En algunos estudios se ha demostrado una disminución de la adherencia al tratamiento por parte del paciente recluso, toda vez que el individuo infectado es liberado, sin completar el tratamiento, y posiblemente este no buscará un centro de salud para dar continuidad al tratamiento (17).

La baja detección de pacientes es uno de los problemas más comunes en muchos municipios del país, hecho que amerita la búsqueda activa de sintomáticos respiratorios, especialmente en lugares con alto índices de tuberculosis, asentamientos donde algunos factores asociados a la proliferación de la bacteria tales como el hacinamiento, la ventilación inadecuada, abuso en el consumo de drogas, desnutrición, la alta proporción de habitantes de minorías étnicas y raciales (frecuentemente inmigrantes de zonas endémicas o de alto riesgo) la escasez de recursos difícilmente permite acceder a los servicios de salud y contribuyen significativamente al aumento en el número de casos $(18,19)$. Esta diversidad geográfica no solo se puede presentar en actividades laborales, agropecuarias y turísticas sino también por convivencia por largo tiempo en lugares como hoteles, fincas cafeteras y centros de reclusión.

El hallazgo de pacientes con baciloscopia negativa y cultivo positivo resalta la importancia de emplear ambos métodos para aumentar la captación de pacientes positivos para TB, ya que el cultivo es una técnica más sensible que la baciloscopia y posibilita el diagnostico de cerca del $90 \%$ de los casos (20). No obstante, dado que aproximadamente entre el 20 y el $50 \%$ de los pacientes con tuberculosis pulmonar son negativos a la baciloscopia y el $10 \%$ de estos son negativos también al cultivo (20) se observa la necesidad de incorporar métodos diagnósticos más sensibles y de caracterización molecular de los aislados (21,22), lo cual permitiría la realización de un diagnostico rápido, una intervención oportuna y precisa. Así mismo, el empleo de estos métodos moleculares permite generar información epidemiológica de gran aplicación al momento de diseñar nuevas estrategias locales y regionales mejorando la efectividad de los programas de control. En la mayor parte del país no se cuenta con las herramientas diagnos- 
ticas necesarias que brinden información valiosa y que puedan contribuir en la toma de medidas para cortar la o las cadenas de transmisión (23).

La aplicación de la técnica de Spoligotyping, permitió determinar la presencia de cuatro genotipos diferentes lo cual indica que el programa de control no ha tenido un impacto significativo en la cadena de transmisión, estos resultados podrían ser comparables a los observados en otras ciudades donde la diversidad de genotipos es mayor en la población urbana respecto a la del área rural por la inestabilidad demográfica generada por constantes migraciones tal como suele ocurrir en la región cafetera $(24,25)$. Lo anterior pone de relieve la importancia de la genotipificación de los aislados de Mycobacterium tuberculosis como herramienta para evaluar la eficacia del programa de control de la tuberculosis a nivel municipal y regional.

La sintomatología predominante en la población infectada por TB fue la persistencia en la tos y expectoración por más de 15 días lo cual representa un riesgo potencial para las personas que conviven con estos individuos, sí se tiene en cuenta el potencial bacilífero y su impacto en la cadena de transmisión, más aun si en sus hogares existen los factores de riesgo que pueden contribuir a su diseminación. Entre los datos registrados, se observo que la población más afectada corresponde a personas de bajos ingresos económicos, lo cual se estableció a través de la vinculación al sistema general de seguridad social en salud nacional, ello representa un impacto negativo en el núcleo familiar y del enfermo, dado que esta situación se manifiesta en incapacidad laboral y una posible emergencia sanitaria por el surgimiento de brotes entre los contactos y de este modo generando un mayor deterioro de la condición socioeconómica y de la salud pública regional (26,27). La incidencia de TB en el municipio de Calarcá genera también un impacto económico, como se evidencia en la población más afectada, perteneciente a las personas en edades económicamente productivas; de igual manera lo representa para las instituciones de salud de tercer nivel como el Hospital departamental, en el cual se captaron y hospitalizaron pacientes no diagnosticados oportunamente, con sintomatología crónica y por ende en condiciones de salud más precarias.

Se hace evidente la necesidad de fortalecer el programa de detección temprana, diagnostico, control y seguimiento de los pacientes tuberculosos en el municipio de Calarcá, mediante el diseño de estrategias más eficientes de búsqueda activa, el mejoramiento en la calidad de la información registrada del paciente y la implementación de medidas terapéuticas adecuadas en tiempo y en acción antibiótica (23). 
Limitaciones del estudio

En el estudio se presentaron algunas limitaciones tales como: toma de las tres muestras el mismo día en razón de las dificultades logísticas y de seguridad en la prisión municipal, lo cual impidió hacer una preparación previa para los pacientes para obtener secreciones bronquiales y no salivales. La localización y continuidad de los pacientes en el estudio se vio afectada por fenómenos migratorios de distinta causa. Finalmente, como se menciono en resultados sólo se pudieron genotipificar cinco aislamientos viables

Agradecimientos. A la Secretaria de Salud del municipio de Calarcá por la financiación del estudio mediante el convenio 099 suscrito entre la Universidad del Quindío y la mencionada institución. A las doctoras Martha Inírida Guerrero y Clara Inés León por su invaluable apoyo en la transferencia científica y técnica. Al Doctor Santiago Fadul Pérez de FUNDASALUD Barranquilla-Atlántico-Colombia por la revisión y aportes al manuscrito, a Ana Cecilia López por su colaboración en el suministro de datos geográficos e historias de los pacientes. A Vilma Audrito Neder presidenta y al personal voluntario de la Liga Antituberculosa del Quindío, por su invaluable apoyo en la orientación y asistencia en la búsqueda activa de pacientes.

\section{REFERENCIAS}

1. World Health Organization Guidelines for implementing collaborative TB and VIH programme activities. Stop TB department of TB and VIH/AIDS; 2005. p. 13-27.

2. Chaparro PE, García I, Guerrero MI, León Cl. Situación de la tuberculosis en Colombia 2002. Biomédica 2004; 24: 102-114.

3. Instituto Seccional de Salud del Quindío Indicadores básicos en salud. SIVIGILA; 2005. p.24.

4. Zuluaga L, Betancur C, Abaunza M, Londoño J. Prevalencia de tuberculosis y enfermedad respiratoria en personas mayores de 15 años de la comuna nororiental de Medellín, Colombia. Bol Of San Panam 1993; 111: 406-413.

5. García I, De la Hoz F, Reyes Y, Montoya P, Guerrero MI, León Cl. Prevalencia de sintomáticos respiratorios, de infección y enfermedad tuberculosa y factores asociados: estudio basado en población, Mitú, Vaupés, 2001. Biomédica 2004; 24 (Supl.): 124-131.

6. Arévalo MY, Dueñas MY. Evaluación del programa intensificado de búsqueda de casos de tuberculosis pulmonar en la comunidad U`wa del municipio de Cubará Boyacá, entre 1 de julio de 2001 y 30 de junio de 2002. Proyección Universitaria. 2003; 23:.3-23.

7. Henao G, De la Hoz F, León Cl, Ribón W, Guerrero MI. Epidemiología clásica y molecular de la tuberculosis en el Guaviare 1997-1998. Informe Quincenal Epidemiológico Nacional 1999; 4: 85-91.

8. García I, Garzón C, Llerena C, Naranjo N, Chaparro P, Moreno E. Panorama de la tuberculosis en Colombia. Informe Epidemiológico Nacional 2003; 8: 369-380.

9. Small MS, Hopewell PC, Singh SP, Paz A, Parsonnet J, Ruston DC, Schecter GF, Daley CL, Schoolnick GK. The Epidemiology of Tuberculosis in San Francisco. APopulation-Based Study Using Conventional and Molecular Methods. N. Engl J Med 330: 1703-1709.

10. Centers for Disease Control and Prevention. Database and statistics software for public health professionals. Epi Info version 4. Atlanta, USA; 2002. 
11. Instituto Nacional de Salud. Bacteriología del Mycobacterium tuberculosis y de Micobacterias no tuberculosas. Manual de procedimientos. Subdirección de epidemiología y laboratorio nacional de referencia. Laboratorio de micobacterias. Bogotá; 2001.

12. Instituto Nacional de Salud de Colombia. Laboratorio de micobacterias. Manual de Tipificación Molecular del complejo Mycobacterium tuberculosis. Subdirección de epidemiología y laboratorio nacional de referencia. Laboratorio de micobacterias. Bogotá, Colombia; 2004. p. 50.

13. Kamerbeek J, Schouls L, Kolk A, Van Agterveld M, Van Soolingen D, Kuijper S, Bunschoten A, Molhuizen H, Shaw R, Goyal M, Van Embden J. Simultaneus detection and strain differentiation of Mycobacterium tuberculosis for diagnosis and epidemiology. J Clin Microbiol 1997; 35: 907-914.

14. Brudey K, Driscoll JR, Rigouts L, Prodinger MW, Gori A, Al-Hajoj SA, Allix C, et al. Mycobacterium tuberculosis complex genetic diversity: mining the fourth international spoligotyping database (SpolDB4) for classification, population genetics and epidemiology. BMC Microbiol, 2006; 6:23.

15. Thorson A, Hoa NP, Long NH. Health-seeking behaviour of individuals with a cough of more than 3 weeks. Lancet 2000; 356:1823-1824.

16. Steenland K, Levine AJ, Sieber K, Schulte P, Aziz D. Incidence of tuberculosis infection among New York State prison employees. Am J Public Health 1997; 87: 2012-2014.

17. Reyes H, Coninx R. Pitfalls of tuberculosis programmes in prisons. Br Med J 1997; 315:14471450.

18. Centers for Disease Control and Prevention. Prevention and control of tuberculosis in correctional facilities: recommendations of the Advisory Council for the Elimination of Tuberculosis. Morbidity and Mortality Weekly Report.1996; 45: 1-27.

19. Chaves F, Dronda F, Cave MD, Alonso-Sanz M, González-López A, Eisenach KD, Ortega A, López-Cubero L, Fernández-Martín I, Catalán S, Bates JH. A longitudinal study of transmission of tuberculosis in a large prison population. Am J Resp Crit Care Med 1997; 155:719-725.

20. Dye C, Scheele S, Dolin P, Pathania V, Raviglione M. Global burden of tuberculosis: estimated incidence, prevalence and mortality by country. JAMA. 1999; 282: 677-686.

21. Sarmiento OL, Weigle KA, Alexander J, Weber DJ, Miller WC. Assessment by meta-analysis of PCR for diagnosis of smear-negative pulmonary tuberculosis. J Clin Microbiol 2003; 41: 3233-3240.

22. Guevara A, Juárez A, Zenteno R. Tuberculosis y la importancia de incorporar nuevas metodologías diagnosticas. MedUNAB. 2003; 6: 46-51.

23. Coronado SM, Cuervo LI, Arenas NE, Torres E, Durango CJ, Castro CM, Guerrero MI, León Cl, Gómez A. Genotipificación por spoligotyping de cepas de Mycobacterium tuberculosis circulantes en el departamento del Quindío durante los años 2003-2005. Infectio 2006; 10: 120.

24. Sola C, Ferdinand S, Mammina C, Nastasi A, Rastogi N. Genetic Diversity of Mycobacterium tuberculosis in Sicily Based on Spoligotyping and Variable Number of Tandem DNA Repeats and Comparison with a Spoligotyping Database for Population-Based Analysis. J Clin Microbiol. 2001; 39 (4) 1559-1565.

25. Braden CR, Templeton GL, Cave MD, Valway S, Onorato IM, Castro KG, et al.. Interpretation of restriction fragment length polymorphism analysis of Mycobacterium tuberculosis isolates from a state with a large rural population. J. Infect. Dis. 1997; 175:1446-1452.

26. Díaz ML, Muñoz S, García LB. Tuberculosis en el hospital universitario San José, Popayán, 1998-2000. Biomédica 2004; 24 (Supl. 25): 92-101.

27. Salazar CL, Uribe MP, Zuluaga WA, Ríos JF, Montes F. Factores de riesgo para el abandono del tratamiento contra la tuberculosis en la ciudad de Medellín. Enero 2000-Junio 2001. CES Medicina 2004; 18: 25-33. 